\title{
Malignant Enteroglucagonoma
}

National Cancer Institute

\section{Source}

National Cancer Institute. Malignant Enterog/ucagonoma. NCI Thesaurus. Code C65191.

A glucagon-producing malignant endocrine neoplasm. It displays vascular invasion and metastasizes to other anatomic sites. 